\title{
A Retrospective Propensity Score Matched Analysis Reveals Superiority of Hypothermic Machine Perfusion over Static Cold Storage in Deceased Donor Kidney Transplantation
}

\author{
Silvia Gasteiger ${ }^{1}$, Valeria Berchtold ${ }^{1}$, Claudia Bösmüller ${ }^{1}$, Lucie Dostal ${ }^{2}$, Hanno Ulmer ${ }^{2} \mathbb{1}$, \\ Christina Bogensperger ${ }^{1}$, Thomas Resch ${ }^{1}$, Michael Rudnicki ${ }^{3}$, Hannes Neuwirt ${ }^{3}$, \\ Rupert Oberhuber ${ }^{1}$, Benno Cardini ${ }^{1}$, Stefan Scheidl ${ }^{1}$, Gert Mayer ${ }^{3}$, Dietmar Öfner ${ }^{1}$, \\ Annemarie Weissenbacher ${ }^{1, *, \dagger}$ and Stefan Schneeberger $1, *, \dagger$ \\ 1 Department of Visceral, Transplant and Thoracic Surgery, Medical University of Innsbruck, \\ 6020 Innsbruck, Austria; silvia.gasteiger@i-med.ac.at (S.G.); valeria.berchtold@i-med.ac.at (V.B.); \\ claudia.boesmueller@tirol-kliniken.at (C.B.); christina.bogensperger@tirol-kliniken.at (C.B.); \\ t.resch@tirol-kliniken.at (T.R.); rupert.oberhuber@i-med.ac.at (R.O.); benno.cardini@i-med.ac.at (B.C.); \\ stefan.scheidl@tirol-kliniken.at (S.S.); dietmar.oefner-velano@tirol-kliniken.at (D.Ö.) \\ 2 Department of Medical Statistics, Informatics and Health Economics, Medical University of Innsbruck, \\ 6020 Innsbruck, Austria; Lucie.Dostal@i-med.ac.at (L.D.); hanno.ulmer@i-med.ac.at (H.U.) \\ 3 Department of Internal Medicine IV, Nephrology and Hypertension, Medical University of Innsbruck, \\ 6020 Innsbruck, Austria; michael.rudnicki@tirol-kliniken.at (M.R.); hannes.neuwirt@tirol-kliniken.at (H.N.); \\ gert.mayer@tirol-kliniken.at (G.M.) \\ * Correspondence: annemarie.weissenbacher@i-med.ac.at (A.W.); stefan.schneeberger@i-med.ac.at (S.S.) \\ + They contributed equally to this work.
}

Received: 23 June 2020; Accepted: 17 July 2020; Published: 21 July 2020

\begin{abstract}
Hypothermic machine perfusion (HMP) has been introduced as an alternative to static cold storage (SCS) in kidney transplantation, but its true benefit in the clinical routine remains incompletely understood. The aim of this study was to assess the effect of HMP vs. SCS in kidney transplantation. All kidney transplants performed between 08/2015 and 12/2019 $(n=347)$ were propensity score (PS) matched for cold ischemia time (CIT), extended criteria donor (ECD), gender mismatch, cytomegalovirus (CMV) mismatch, re-transplantation and Eurotransplant (ET) senior program. A total of 103 HMP and 103 SCS instances fitted the matching criteria. Prior to PS matching, the CIT was longer in the HMP group ( $17.5 \mathrm{~h}$ vs. $13.3 \mathrm{~h} ; p<0.001)$, while the delayed graft function (DGF) rates were $29.8 \%$ and $32.3 \%$ in HMP and SCS, respectively. In the PS matched groups, the DGF rate was $64.1 \%$ in SCS vs. $31.1 \%$ following HMP: equivalent to a $51.5 \%$ reduction of the DGF rate (OR $0.485,95 \%$ CI $0.318-0.740)$. DGF was associated with decreased 1- and 3-year graft survival $(100 \%$ and $96.3 \%$ vs. $90.8 \%$ and $86.7 \%, p=0.001$ and $p=0.008)$ or a 4.1 -fold increased risk of graft failure ( $\mathrm{HR}=4.108 ; 95 \% \mathrm{CI}: 1.336-12.631 ; p=0.014)$. HMP significantly reduces DGF in kidney transplantation. DGF remains a strong predictor of graft survival.
\end{abstract}

Keywords: kidney transplantation; hypothermic machine perfusion; delayed graft function

\section{Introduction}

Kidney transplantation (KTx) is the therapy of choice for patients with end-stage renal disease (ESRD). The shortage of donor organs results in an ever-increasing waiting list and accumulating patient deaths. The donor pool and organ utilization have been expanded in recent years through the use of an increased number of marginal organs [1]. Extended criteria donor (ECD) organs and organs 
recovered from donation after circulatory death (DCD), however, are more vulnerable to ischemia reperfusion injury. This eventually translates into higher rates of delayed graft function (DGF), primary non-function (PNF) and inferior graft survival [2-5]. While ECD kidneys have a 1.7 times greater risk for graft failure [6,7], recipients still gain a significant survival benefit compared to dialysis [8]. Nevertheless, ECD organs with prolonged travel time are discarded at a high rate, since transplant surgeons and physicians fear the increased risk of DGF and PNF [1]. Considering the benefit of ECD organ use and further decreasing the discard rate, optimal organ preservation and ex situ organ quality assessment remain key tools for eventually increasing utilization in kidney transplantation.

Early attempts of dynamic organ preservation in the 1960s $[9,10]$ were not pursued further due to technical and logistical hurdles. The technology was reintroduced decades later and a prospective randomized trial by Moers et al indicated that DGF rates could be lowered by hypothermic machine perfusion (HMP) compared to static cold storage (SCS) [11]. Superior outcomes and/or preferable effects on kidney allografts and transplant outcomes have been confirmed by others [12-14]. The positive impact seems to be more pronounced in ECD kidneys and is presumed to result from the protective effect on the endothelium and the removal of waste products [15]. Since the benefit was not profound in some trials and not uniformly considered to outweigh the logistics and costs involved, SCS largely remains the current standard of kidney preservation [16]. The aim of our study was to investigate the impact of HMP on kidney graft function after deceased donor kidney transplantation in an HMP cohort propensity score matched with SCS (PS cohort).

\section{Material and Methods}

Following institutional ethics board approval (study number 1246/2019), a retrospective analysis of all deceased donor KTx performed at the Department of Visceral, Transplant and Thoracic Surgery, Medical University of Innsbruck, between August 2015 and December 2019, was conducted. Patients had undergone either single or double KTx from deceased donors. The LifePort Kidney Transporter ${ }^{\circledR}$ (Organ Recovery Systems, Itasca, IL, USA) and UW Machine Perfusion Solution (Belzer MPS ${ }^{\circledR}$ ) were used for HMP. Before undergoing HMP, every kidney experienced a period of SCS. Kidneys from DCD were excluded since they represent only $1 \%$ of kidneys in our cohort. En-bloc KTx from pediatric donors, simultaneous pancreas kidney transplants (SPK), combined liver and kidney transplants (LKTx) and combined heart and kidney transplants (HKTx) were also excluded. The study was carried out in accordance with the STROBE (Strengthening The Reporting of OBservational Studies in Epidemiology) checklist [17]. The decision whether a kidney underwent HMP or SCS was based on the availability of immediate operation room (OR) capacity and crossmatch results.

Kidney graft loss was defined as return to chronic dialysis. Graft survival was not censored for death. DGF was defined as the need for at least one dialysis within the first seven days after transplant with the exception of dialysis for hyperkalemia or hypervolemia within the first 12 hours post-transplant [18]. Cytomegalovirus (CMV) mismatch was defined as CMV IgG positive donor to CMV IgG negative recipient ( $\mathrm{D}+\mathrm{R}-$ ). "Daytime" procedure was defined as transplantations with skin incision between $8 \mathrm{AM}$ and $8 \mathrm{PM}$, "Nighttime" transplants were defined by skin incision between $8 \mathrm{PM}$ and 8 AM [19]. ECD was defined as age $\geq 60$ years, or age of 50 to 59 years with two of the following: a history of hypertension, a creatinine greater than or equal to $1.5 \mathrm{mg} / \mathrm{dl}$ or death resulting from cardiovascular accident as defined in 2002 by Port et al [20].

Standard immunosuppression included induction therapy with $20 \mathrm{mg}$ basiliximab for first-KTx recipients (day 0 and 3); tacrolimus was administered from the first postoperative day onwards, aiming for trough levels of $8-10 \mathrm{ng} / \mathrm{mL}$ during the first three months, according to our institutional standard; mycophenolate mofetil $1000 \mathrm{mg}$ was given twice daily together with a steroid taper. Patients undergoing re-transplantation received a single dose of $8 \mathrm{mg} / \mathrm{kg}$ antithymocyte globulin (ATG) as induction therapy. For infection prophylaxis, ampicillin/sulbactam was administered for three days. In case of CMV mismatch ( $\mathrm{D}+/ \mathrm{R}-)$ or induction therapy with ATG, an antiviral prophylaxis with 
valganciclovir was applied for 90 days. Patients with DGF received neither additional treatment (i.e. additional induction therapy) nor standardized protocol biopsies.

\section{Statistical Analysis}

Continuous variables are presented as mean \pm standard deviation (SD) in case of a normal distribution and as median and range otherwise. Categorical data are presented as number of cases with percentage.

Baseline characteristics between kidney preservation (HMP/SCS) were tested using the independent-sample Mann-Whitney U test for non-normally distributed continuous variables and $\chi^{2}$-test or Fisher's exact test for categorical variables, as appropriate. Standardized mean differences [21] for comparing means and prevalence between groups are reported.

A propensity score matching with caliper of 0.05 was performed based on following variables: donor type (SCD/ECD), CIT, gender and CMV mismatch, number of transplants (first or Re-Tx) and ET-senior program [22]. The quality of matching was assessed by calculation of the standardized mean difference (SMD) between selected variables, with a SMD $<0.10$ reflecting good matching [21].

The effect of HMP on the incidence of DGF was modelled with the conditional logistic regression analysis. Patient and graft survival were calculated with Kaplan-Meier survival analysis method. Kaplan-Meier curves were limited at a number at risk of $10 \%$ of patients. The effect of DGF on graft survival was assessed using Cox regression analysis.

All analyses were performed with SPSS (Statistical Package for the Social Sciences) version 25 (IBM; Armonk, NY, USA). A $p$-value of less than 0.05 was considered to indicate statistical significance.

\section{Results}

In total, 347 patients were transplanted between August 2015 and December 2019. HMP was applied in $124 / 347$ patients (35.7\%). In the remaining $223 / 347$ patients $(64.3 \%)$, kidneys were transplanted following SCS. DGF occurred in 37/124 patients (29.8\%) and 72/223 patients (32.3\%) following HMP and SCS $(p=0.638)$. Mean CIT was $17.5 \pm 5.1 \mathrm{~h}$ and $13.3 \pm 4.6 \mathrm{~h}$ in the HMP and SCS group, respectively $(p<0.001$, Table 1$)$.

Table 1. Baseline characteristics of the unmatched kidney transplant cohort $(n=347)$.

\begin{tabular}{ccccc}
\hline & HMP, $\boldsymbol{n}=\mathbf{1 2 4}$ & SCS, $\boldsymbol{n}=\mathbf{2 2 3}$ & $\boldsymbol{p}$-Value & SMD \\
\hline CIT (hours) * & $17.5( \pm 5.1)$ & $13.3( \pm 4.6)$ & $p<0.001$ & 0.865 \\
ECD $(n, \%)$ & $61(49.2 \%)$ & $95(42.6 \%)$ & $p=0.237$ & 0.108 \\
SCD $(n, \%)$ & $63(50.8 \%)$ & $128(57.4 \%)$ & $p=0.237$ & 0.108 \\
Gender MM $(n, \%)$ & $66(53.2 \%)$ & $110(49.3 \%)$ & $p=0.486$ & 0.064 \\
CMV MM $(n, \%)$ & $21(16.9 \%)$ & $38(17.1 \%)$ & $p=0.966$ & 0.004 \\
Re-TX $(n, \%)$ & $31(25.0 \%)$ & $44(19.7 \%)$ & $p=0.253$ & 0.103 \\
ET-Senior $(n, \%)$ & $25(20.2 \%)$ & $43(19.3 \%)$ & $p=0.843$ & 0.018 \\
\hline
\end{tabular}

SMD: standardized mean difference, CIT: cold ischemia time, ECD: extended criteria donor, SCD: standard criteria donor, MM: mismatch, TX: transplantation, ET: Eurotransplant. * values are mean (SD).

To correct for key donor and recipient factors and CIT, propensity score matching was performed in the ratio of 1:1 (103 HMP kidneys and 103 SCS kidneys). Figure 1 displays the algorithm of patient selection. Following propensity score matching the CIT was $16.3 \pm 4.3$ and $16.0 \pm 4.4 \mathrm{~h}$ in the HMP-cohort and SCS-cohort, respectively. Baseline characteristics following PS-matching are shown in Table 2, donor and recipient demographics as well as transplant factors (matched cohort) are shown in Table 3. 


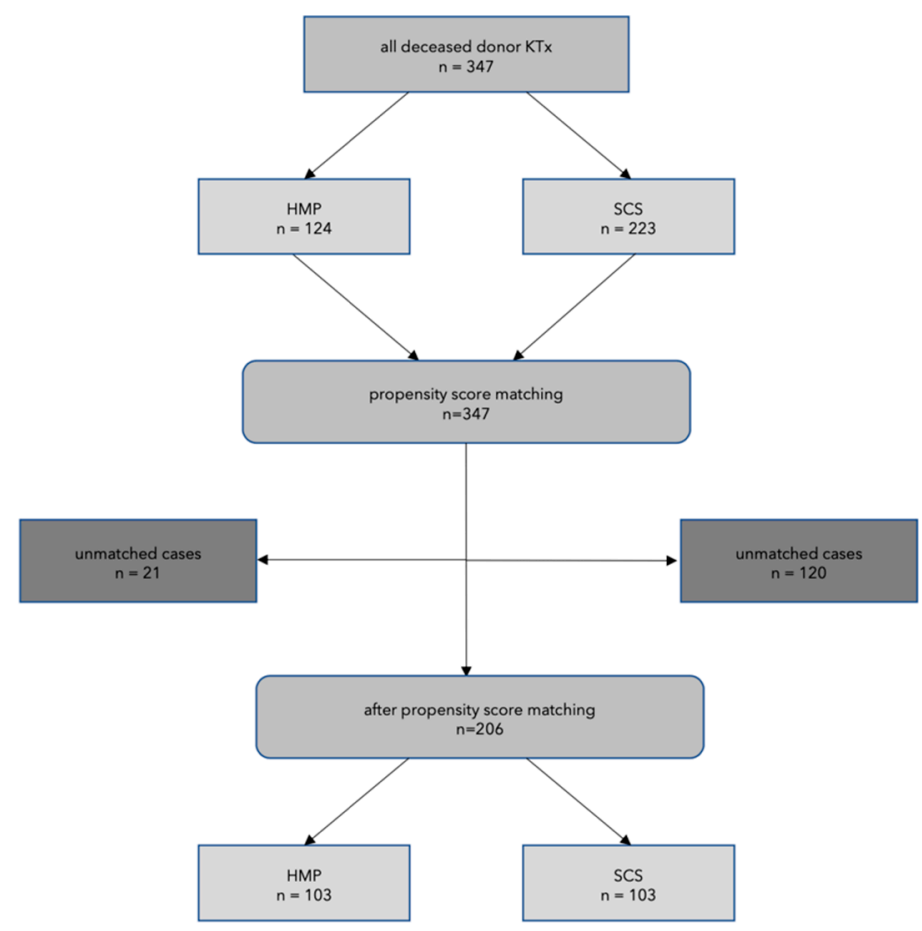

Figure 1. Flow chart of patient selection. HMP: hypothermic machine perfusion, SCS: static cold storage.

Table 2. Baseline characteristics of the propensity-score matched cohort $(n=206)$.

\begin{tabular}{cccc}
\hline & HMP, $n=\mathbf{1 0 3}$ & SCS, $\boldsymbol{n}=\mathbf{1 0 3}$ & SMD \\
\hline CIT (hours) & $16.32( \pm 4.3)$ & $15.97( \pm 4.4)$ & 0.059 \\
ECD $(n, \%)$ & $50(48.5 \%)$ & $55(53.4 \%)$ & 0.080 \\
SCD $(n, \%)$ & $53(51.5 \%)$ & $48(46.6 \%)$ & 0.080 \\
Gender MM $(n, \%)$ & $54(52.4 \%)$ & $49(47.6 \%)$ & 0.078 \\
CMV MM $(n, \%)$ & $17(16.5 \%)$ & $13(12.6 \%)$ & 0.092 \\
Re-TX $(n, \%)$ & $23(22.3 \%)$ & $25(24.3 \%)$ & 0.019 \\
ET-Senior $(n, \%)$ & $19(18.4 \%)$ & $16(15.5 \%)$ & 0.062 \\
\hline
\end{tabular}

SMD: standardized mean difference, CIT: cold ischemia time, ECD: extended criteria donor, SCD: standard criteria donor, MM: mismatch, CMV: cytomegalovirus, Re-TX: re-transplantation, ET: Eurotransplant. * values are mean (SD).

Table 3. Donor and recipient demographics and transplant factors stratified by HMP and SCS.

\begin{tabular}{ccc}
\hline & HMP, $\boldsymbol{n}=\mathbf{1 0 3}$ & SCS, $\boldsymbol{n = 1 0 3}$ \\
\hline Recipient age, median (range) & $57(28-79)$ & $58(26-76)$ \\
Recipient male gender, $n(\%)$ & $74(71.8 \%)$ & $71(68.9 \%)$ \\
Recipient BMI kg/m ${ }^{2}$, mean \pm SD & $25.5 \pm 4.6$ & $26.4 \pm 4.2$ \\
Prior TX, $n(\%)$ & $23(22.3 \%)$ & $2524.3 \%$ \\
Double kidney TX, $n(\%)$ & $6(5.8 \%)$ & $9(8.7 \%)$ \\
Donor age, median (range) & $55(18-82)$ & $55(18-84)$ \\
Donor male gender, $n(\%)$ & $52(50.5 \%)$ & $64(62.1 \%)$ \\
Donor BMI kg/m ${ }^{2}$, mean \pm SD & $26.8 \pm 5.2$ & $27.2 \pm 4.4$ \\
Extended criteria donor, $n(\%)$ & $50(48.5 \%)$ & $55(53.4 \%)$ \\
Kidney donor risk index (KDRI), mean \pm SD & $1.29 \pm 0.42$ & $1.31 \pm 0.42$ \\
Kidney donor profile index (KDPI), mean \pm SD & $66.7 \pm 23.9$ & $67.3 \pm 25.3$ \\
Cause of end stage renal disease & & \\
Glomerulonephritis & $37(35.9 \%)$ & $40(38.8 \%)$ \\
Diabetic nephropathy & $19(18.5 \%)$ & $16(15.5 \%)$ \\
Hereditary renal disease & $12(11.7)$ & $15(14.6 \%)$ \\
Vascular nephropathy & $12(11.7 \%)$ & $13(12.6 \%)$ \\
Others & $23(22.3 \%)$ & $19(18.4 \%)$ \\
\hline
\end{tabular}


Table 3. Cont.

\begin{tabular}{ccc}
\hline & HMP, $\boldsymbol{n}=\mathbf{1 0 3}$ & SCS, $\boldsymbol{n = 1 0 3}$ \\
\hline Cold ischemia time in hours, mean \pm SD & $16.32 \pm 4.3$ & $15.97 \pm 4.4$ \\
Anastomosis time in minutes, mean \pm SD & $33 \pm 10$ & $31 \pm 9$ \\
HLA A mm, mean \pm SD & $1.02 \pm 0.61$ & $0.98 \pm 0.69$ \\
0 and 1 & $83(80.6 \%)$ & $80(77.7 \%)$ \\
2 & $20(19.4 \%)$ & $22(21.3 \%)$ \\
HLA B mm, mean \pm SD & $1.29 \pm 0.69$ & $1.15 \pm 0.66$ \\
0 and 1 & $59(57.3 \%)$ & $72(69.9 \%)$ \\
2 & $44(42.7 \%)$ & $31(30.1 \%)$ \\
HLA DR mm, mean \pm SD & $1.20 \pm 0.63$ & $1.02 \pm 0.71$ \\
0 and 1 & $70(68.0 \%)$ & $76(73.8 \%)$ \\
2 & $33(32.0 \%)$ & $27(26.2 \%)$ \\
\hline
\end{tabular}

BMI: body mass index, SD: standard deviation, TX: transplantation, HLA: human leukocyte antigen.

\subsection{Incidence of DGF}

DGF occurred in 98/206 patients $(47.6 \%)$ and was less frequent in recipients of SCD organs $(n=101)$ compared to $\operatorname{ECD}(n=105) ; 41 / 101(40.6 \%)$ vs. $57 / 105(54.3 \%), p=0.049$. DGF was significantly less frequent following HMP compared to SCS; (32/103 (31.1\%) vs. 66/103 (64.1\%), $p<0.001$, Figure 2A). The difference was significant for both SCD and ECD kidneys: DGF occurred in 13/53 (24.5\%) SCD kidneys compared to 19/50 (38\%) ECD organs in the HMP group. Following SCS, DGF occurred in $28 / 48$ (58.3\%) SCD kidneys, while the DGF rate was 69.1\% (38/55) in ECD kidneys; $p=0.001$ for SCD and ECD (Figure 2B). A conditional logistic regression analysis modelling for the risk of DGF revealed that HMP resulted in a $51.5 \%$ reduction of DGF (OR $=0.485 ; 95 \%$ CI: $0.318-0.740 ; p=0.001)$.
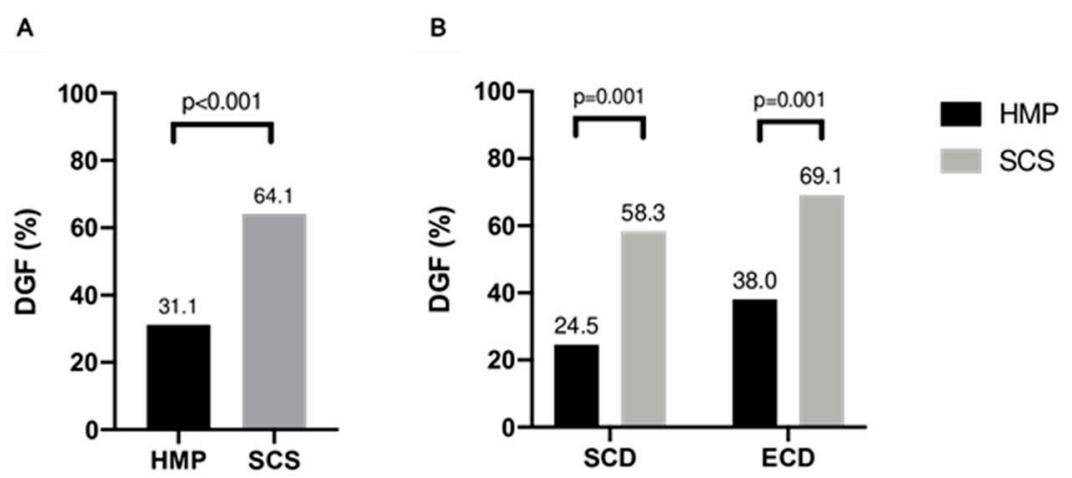

Figure 2. (A) Delayed graft function rate of hypothermically perfused deceased donor kidneys compared to kidneys statically stored on ice; (B) delayed graft function rate of kidneys after hypothermic machine perfusion compared to organs stored on ice stratified for standard and extended criteria donors.

\subsection{Daytime vs. Nighttime Procedures}

When stratifying transplant procedures by time of surgery, 39.3\% $(n=81)$ of transplants were performed during nighttime. SCS kidneys were more likely to be transplanted during nighttime $(56.8 \%$ vs. $43.2 \%, p=0.077$ ) compared to HMP kidneys. Nighttime vs. daytime kidney transplant procedures did not differ in regard to postoperative complication rate (27.2\% vs. $27.2 \%)$, DGF (49.4\% vs. $46.4 \%$ ) and graft loss $(9.5 \%$ vs. $8.8 \%)$.

\subsection{HMP Parameters}

Median HMP time was 6.2 hours (1.9-18.8). Preservation time did not differ between patients with immediate graft function and DGF (6.1 vs. 6.7, $p=0.963)$. The flow increased significantly over the course of HMP (70mL/min (range 6-239) vs. 107mL/min (range 48-240), $p<0.001$ ) and 
the intra-renal-resistance (IRR) index declined over time $(0.39 \mathrm{mmHg} / \mathrm{mL} / \mathrm{min}$ (range 0.6-4.7) vs. $0.22 \mathrm{mmHg} / \mathrm{mL} / \mathrm{min}$ (range 0.1-0.5), $p<0.001$ ). Flow and IRR index at 15 minutes of HMP were $82 \mathrm{~mL} / \mathrm{min}$ (range 6-239) and $0.30 \mathrm{mmHg} / \mathrm{mL} / \mathrm{min}$ (range 0.1-4.66) in patients with an immediate graft function compared to $55 \mathrm{~mL} / \mathrm{min}$ (range 18-191) and $0.46 \mathrm{mmHg} / \mathrm{mL} / \mathrm{min}$ (range 0.1-1.22) in patients with DGF; $p=0.074$ and $p=0.080$, respectively. At the end of perfusion, flow and IRR did not differ among patients with, and without DGF; $(95 \mathrm{~mL} / \mathrm{min}$ vs. $115 \mathrm{~mL} / \mathrm{min}$ and $0.26 \mathrm{mmHg} / \mathrm{mL} / \mathrm{min}$ vs. $0.22 \mathrm{mmHg} / \mathrm{mL} / \mathrm{min}, p=0.172$ and $p=0.130$ ).

\subsection{Complications}

Within the median observational period of 24 months, postoperative complications occurred in 56/206 patients (27.2\%) and were comparable in patients receiving kidneys after HMP 27/103 (26.2\%) and SCS 29/103 (28.2\%). Most common complications were hematoma $(n=14 / 6.8 \%)$, lymphoceles ( $n=19 / 9.2 \%)$, urological complications $(n=15 / 7.3 \%)$, wound infections $(n=13 / 6.3 \%)$ and vascular complications $(n=4 / 1.9 \%)$. Wound infections occurred significantly more often following SCS than after HMP; $10 / 9.7 \%$ vs. $3 / 2.9 \%, p=0.041$. The incidence of all other postoperative complications was comparable between groups as listed in Table 4.

Table 4. Postoperative complications of the propensity-score matched cohort.

\begin{tabular}{lccc}
\hline & HMP, $\boldsymbol{n}=\mathbf{1 0 3}$ & SCS, $\boldsymbol{n = 1 0 3}$ & $\boldsymbol{p}$-Value \\
\hline overall complications & $27(26.2 \%)$ & $29(28.2 \%)$ & 0.438 \\
hematoma & $6(5.8 \%)$ & $8(7.8 \%)$ & 0.392 \\
lymphocele & $6(5.8 \%)$ & $13(12.6 \%)$ & 0.074 \\
urological complications & $8(7.8 \%)$ & $7(6.8 \%)$ & 0.500 \\
$\quad$ urinary leakage & $4(3.9 \%)$ & $3(2.9 \%)$ & \\
ureteral stenosis & $2(1.9 \%)$ & $4(3.9 \%)$ & \\
vesicoureteral reflux & $2(1.9 \%)$ & 0 & 0.041 \\
wound infections & $3(2.9 \%)$ & $10(9.7 \%)$ & 0.500 \\
vascular complications & $4(3.8 \%)$ & $3(2.9 \%)$ & \\
arterial stenosis & $1(1.0 \%)$ & $2(1.9 \%)$ & \\
pseudoaneurysm & $1(1.0 \%)$ & 0 & \\
\hline
\end{tabular}

\subsection{Patient and Graft Survival}

After a median follow-up of 24.2 (range 0.33-53.8) months, 1- and 3-year patient survival was 99.0\% and $97.1 \%$, respectively. The 1- and 3-year graft survival rate reached $95.6 \%$ and $91.8 \%$, respectively.

In the HMP group, 1- and 3-year patient survival was $100 \%$ and $99.0 \%$ and graft survival reached $97.1 \%$ and $95.2 \%$, respectively. Primary non function (PNF) occurred in one patient following HMP. Following SCS, 1- and 3-year patient survival was $99.0 \%$ and $95.2 \%$, whereas graft survival reached $94.2 \%$ and $88.4 \%$, respectively. Patient and graft survival rates at 3-years post-transplant did not differ significantly between HMP and SCS (log rank, $p=0.185$ and $p=0.272$, respectively, Figure 3).

Graft survival was superior in patients with immediate graft function compared to patients with DGF. This difference was statistically significant at both, one and three years post-transplant: in patients with immediate graft function, 1- and 3-year graft survival reached $100 \%$ and $96.3 \%$ compared to $90.8 \%$ and $86.7 \%$ in patients with DGF (log rank, $p=0.001$ and $p=0.008$, respectively, Figure 4 ). 
A

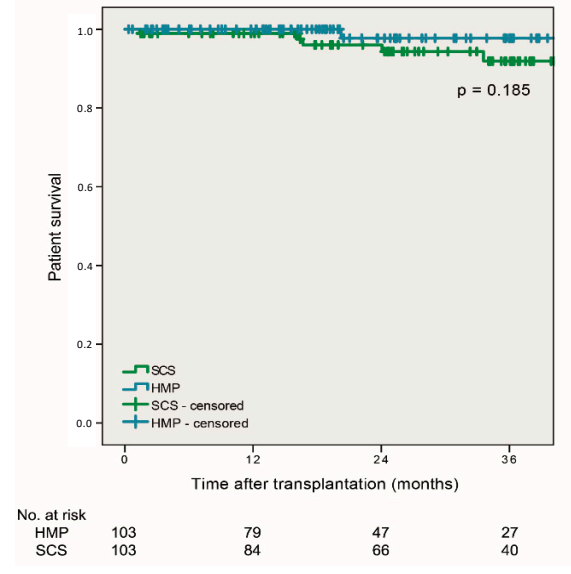

B

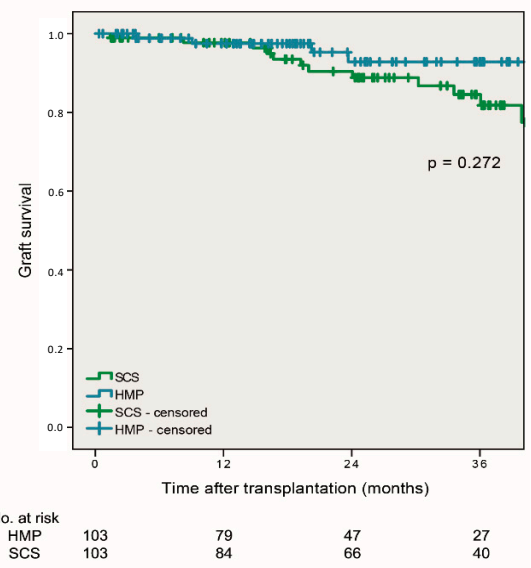

Figure 3. (A) Estimated 3-year patient and (B) 3-year graft survival rates after transplantation of HMP and SCS kidneys.

A

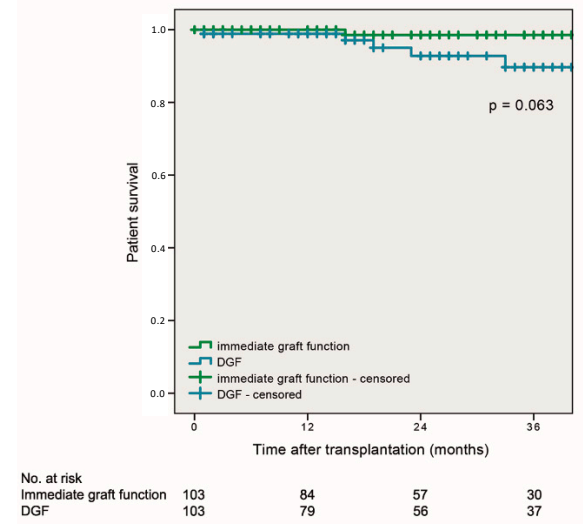

B

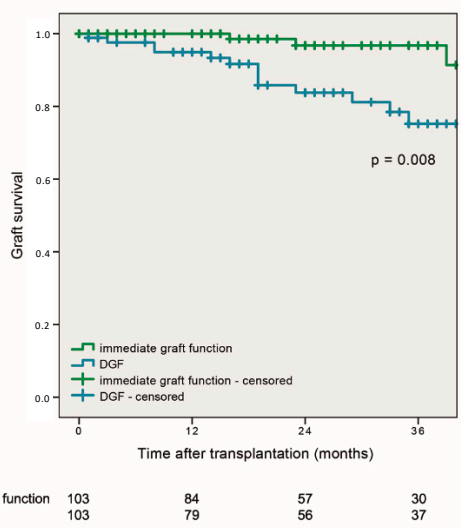

Figure 4. (A) Estimated 3-year patient and (B) 3-year graft survival rates of patients stratified for immediate graft function and delayed graft function.

In a Cox regression analysis, recipients suffering from DGF had a 4.1-times increased risk of graft failure (HR $=4.108 ; 95 \%$ CI: $1.336-12.631 ; p=0.014)$. One year patient survival was $100 \%$ in patients with immediate graft function and $99 \%$ in patients with DGF (log rank, $p=0.296$ ). Patient survival at 3 years after transplantation was superior in cases with immediate graft function vs. DGF, but the difference did not reach statistical significance $(99.1 \%$ vs. $94.9 \%$; log rank, $p=0.063)$.

In patients receiving a kidney after HMP, the glomerular filtration rate (GFR) at last follow-up was $44.8 \mathrm{~mL} / \mathrm{min} / 1.73 \mathrm{~m}^{2}$ (range 11.3-122.4) compared to $39.0 \mathrm{~mL} / \mathrm{min} / 1.73 \mathrm{~m}^{2}$ (range 16.7-98.8) in patients after transplanting an SCS organ; $(p=0.177)$. Serum creatinine at last follow-up was $1.54 \mathrm{mg} / \mathrm{dL}$ (range $0.5-4.14$ ) after HMP and $1.61 \mathrm{mg} / \mathrm{dL}$ (range 0.8-3.80) in patients after SCS $(p=0.260)$.

Serum creatinine and GFR at last follow-up were inferior in patients with DGF: $1.92 \mathrm{mg} / \mathrm{dL}$ (range $0.9-4.14$ ) vs. $1.44 \mathrm{mg} / \mathrm{dL}$ (range $0.5-3.9$ ) and $33.9 \mathrm{~mL} / \mathrm{min} / 1.73 \mathrm{~m}^{2}$ (range $11.3-82.0$ ) vs. $45.9 \mathrm{~mL} / \mathrm{min} / 1.73$ $\mathrm{m}^{2}$ (range 15.6-122.4); $p<0.001$ for both, Figure 5. This difference was also evident in subgroup analysis (HMP vs. SCS, see Table 5). Serum creatinine and GFR at last follow-up were also inferior in patients who received an ECD kidney compared to those who received a SCD organ: $1.85 \mathrm{mg} / \mathrm{dL}$ (range $0.5-3.9$ ) vs. $1.37 \mathrm{mg} / \mathrm{dL}$ (range $0.7-4.1$ ) and $34.5 \mathrm{~mL} / \mathrm{min} / 1.73 \mathrm{~m}^{2}$ (range $15.6-122.4$ ) vs. 51.7 $\mathrm{mL} / \mathrm{min} / 1.73 \mathrm{~m}^{2}$ (range 11.3-99.4); $p<0.001$ for both. 
A

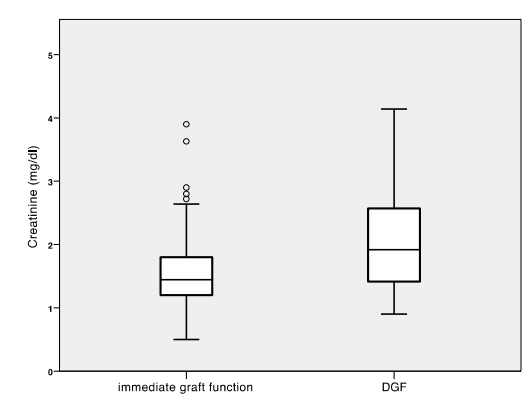

B

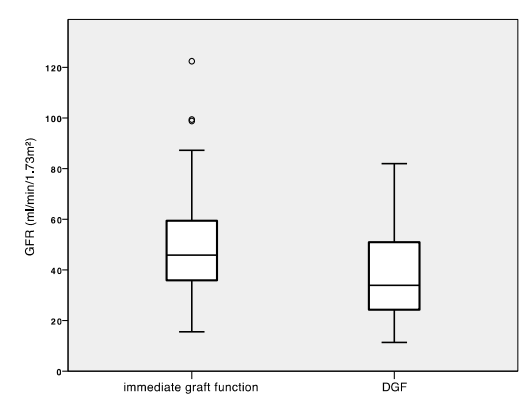

Figure 5. (A) Median serum creatinine and (B) estimated glomerular filtration rate (MDRD) after a median follow-up of 24 months for patients with immediate and delayed graft function. ${ }^{\circ}$ statistical outliers.

Table 5. Subgroup analysis of serum creatinine and eGFR in patients with immediate graft function and DGF.

\begin{tabular}{lccc}
\hline & DGF & No DGF & $p$-Value \\
\hline HMP & & & \\
$\quad$ serum creatinine & $2.0(0.9-4.1)$ & $1.42(0.5-3.9)$ & 0.001 \\
GFR & $32.9(11.3-76.3)$ & $46.8(15.6-122.4)$ & 0.004 \\
SCS & & & \\
$\quad$ serum creatinine & $1.84(0.92-3.8)$ & $1.5(0.8-3.63)$ & 0.009 \\
$\quad$ GFR & $33.9(16.7-82.0)$ & $42.2(19.6-98.8)$ & 0.015 \\
\hline
\end{tabular}

GFR: glomerular filtration rate; values are median (range).

\section{Discussion}

The present study assesses the outcome after deceased donor kidney transplantation in organs undergoing HMP compared to a propensity score matched SCS cohort. Our analysis reveals a relatively strong clinical benefit of HMP with significant lower DGF rates in both ECD and SCD kidneys. However, the novel and important finding of our study is the fact that we can operate in a back-to-base fashion, accepting SCS kidneys and connecting them to HMP as soon as the organs arrive at our center. To our knowledge, our study describes for the first time that now a similar beneficial result with HMP over SCS can be achieved with SCS and end-HMP for 6.2 hours. Boffa et al presented the POMP trial by the COPE consortium [23] fairly recently at the congress of the British Transplant Society. In their prospective trial, the combination of SCS and oxygenated end-HMP in ECD kidneys did not result in any improvement of outcomes, despite shorter CIT compared to our cohort and an HMP time of 4.7 hours. Given the fact that 6.2 hours in our cohort seemed to have a beneficial effect, it will require more investigational procedures to detect the mechanism of this very impact and, if possible, to define a certain time frame of SCS and HMP leading to a favorable outcome.

Delayed graft function is the most significant early complication after deceased donor kidney transplantation. Further to its immediate effect on patient wellbeing and hospital stay, DGF has a negative long term impact on graft and patient survival [24]. The incidence of DGF ranges from $20 \%$ to $70 \%$ and it differs significantly between regions and kidney transplant programs [25]. In several randomized controlled trials, HMP has been shown to decrease DGF rates and to boost graft survival, especially in ECD and DCD kidneys [12,26,27]. In the unmatched comparison of this trial, the DGF rate was comparable between HMP and SCS (29.8\% following HMP and 32.3\% in SCS). The CIT was significantly longer in the HMP group. In this regard, our study suggests that HMP may allow safe prolongation of CIT. To eliminate heterogeneity between the two cohorts, we performed propensity score matching based for key donor and recipient factors and CIT. This approach revealed that HMP was associated with a $51.5 \%$ reduction of the DGF rate $(p=0.001)$ in our cohort. While the protective 
effect of HMP is presumably more pronounced in ECD kidneys [4,28], we found no difference between SCD and ECD kidneys. This is in line with previously published data by Moers et al, suggesting that HMP has a beneficial effect on the short-term outcome in all types of deceased-donor kidney transplants [11]. Interestingly, the positive effect of HMP persisted despite a long CIT (16 hours). This is in contrast to data published by Kox et al who stated that organs with the shortest CIT benefitted the most, whereas the DGF rate in kidneys with over 10 hours of CIT did not differ between HMP and SCS [29].

Graft survival at three years reached 91.8\% (95.2\% following HMP and 88.4\% following SCS). In our series, the difference in graft survival between HMP and SCS did not reach statistical significance. With the apparent trend, we believe that this is due to the small sample size and reference properly powered studies (e.g., the Eurotransplant trial [2]), which reported a graft survival benefit after one and three years following HMP. In our cohort, DGF was associated with a 4.1-times increased risk for graft failure, resulting in a graft survival rate of only $86.7 \%$ at three years (compared to $96.3 \%$ in non DGF kidneys). Hence, the effect of HMP in kidney transplantation seems significant and puts into question why HMP has not evolved as the new standard of care with more widely adoption.

Moreover, HMP led to a shift towards more daytime kidney transplant procedures. The impact of the daytime of surgery on the outcomes after transplantation is contradictory. Fechner et al observed a higher incidence of complications as well as inferior graft survival following nighttime kidney transplant procedures [30]. In our cohort, outcomes were comparable between daytime and nighttime transplants. This is in line with prior published data from our center [19], as well as others [31]. Moving surgical procedures from nighttime to daytime may be particularly beneficial in times of working hour restrictions and staffing shortage. Further to this, safe prolongation of preservation times may facilitate hemodynamic stabilization in delayed kidney-liver transplantation and delayed kidney-heart transplantation or allow for pretreatment of the recipient, e.g., immunoadsorption and other desensitization strategies.

Most studies performed in the last decade used the LifePort Kidney Transporter ${ }^{\circledR}$. Further to the established HMP techniques, oxygenation during HMP has shown beneficial effect in several preclinical studies [32-34]. First clinical data from a double blinded, randomized, paired phase 3 trial (COPE trial) are promising, suggesting that oxygenated HMP improves graft function at one year compared to non-oxygenated HMP [35]. These findings need further confirmation, but it is reasonable to assume that oxygenated HMP will eventually be implemented and improve the outcome after kidney transplantation further.

Poor kidney function in the first week is detrimental for both the patients' perception and the longevity of the deceased donor kidney. Hypothermic machine perfusion was found to be clearly beneficial in several randomized clinical trials. We herein provide evidence that the actual benefit of HMP in the real-world scenario might be more significant and impactful than expected. Hypothermic machine perfusion impressively halved the DGF rate compared to SCS kidneys after similar periods of CIT. For the purpose of achieving the most optimal kidney preservation, HMP should be the preferred technique. Moreover, SCS kidney transportation in the ice box is relatively cheap, but if the back-to-base approach is used and the recipient center based HMP allows to achieve a beneficial effect, this could be very attractive and still cost-effective, especially within an international long-distance exchange organization. Future studies should emphasize on the impact of different durations of CIT accompanied by short periods of HMP to detect possible "rescue" time frames to consider even short periods of end ischemic HMP, for example, 2-3 hours only, crucial for optimal outcome after deceased donor kidney transplantation.

Author Contributions: Conceptualization, G.M., D.Ö., A.W. and S.S. (Stefan Schneeberger); Data curation, S.G., V.B., C.B. (Claudia Bösmüller), C.B. (Christina Bogensperger), T.R., M.R., H.N., R.O., B.C. and S.S. (Stefan Scheidl); Formal analysis, S.G., L.D. and H.U.; Investigation, S.G.; Methodology, L.D., H.U., R.O., B.C. and S.S. (Stefan Scheidl); Project administration, A.W. and S.S. (Stefan Schneeberger); Software, L.D. and H.U.; Supervision, T.R., R.O., B.C. and S.S. (Stefan Scheidl); Validation, A.W. and S.S. (Stefan Schneeberger); Writing—original draft, S.G.; Writing-review \& editing, V.B., C.B. (Claudia Bösmüller), C.B. (Christina Bogensperger), T.R., M.R., H.N., R.O., 
B.C., S.S. (Stefan Scheidl), G.M., D.Ö., A.W. and S.S. (Stefan Schneeberger). All authors have read and agreed to the published version of the manuscript.

Funding: This research received no external funding.

Conflicts of Interest: The authors declare no conflict of interest.

\section{References}

1. Aubert, O.; Reese, P.P.; Audry, B.; Bouatou, Y.; Raynaud, M.; Viglietti, D.; Legendre, C.; Glotz, D.; Empana, J.-P.; Jouven, X.; et al. Disparities in Acceptance of Deceased Donor Kidneys Between the United States and France and Estimated Effects of Increased US Acceptance. JAMA Intern. Med. 2019, 179, 1365. [CrossRef] [PubMed]

2. Jochmans, I.; Moers, C.; Smits, J.M.; Leuvenink, H.G.; Treckmann, J.; Paul, A.; Rahmel, A.; Squifflet, J.-P.; Van Heurn, E.; Monbaliu, D.; et al. Machine perfusion versus cold storage for the preservation of kidneys donated after cardiac death: A multicenter, randomized, controlled trial. Ann Surg. 2010, 252, 756-764. [CrossRef]

3. van der Vliet, J.A.; Warlé, M.C.; Cheung, C.L.; Teerenstra, S.; Hoitsma, A.J. Influence of prolonged cold ischemia in renal transplantation. Clin. Transplant. 2011, 25, E612-E616. [CrossRef] [PubMed]

4. Jochmans, I.; O'Callaghan, J.M.; Pirenne, J.; Ploeg, R.J. Hypothermic machine perfusion of kidneys retrieved from standard and high-risk donors. Transpl. Int. 2015, 28, 665-676. [CrossRef] [PubMed]

5. Helfer, M.S.; Pompeo, J.C.; Costa, O.R.S.; Vicari, A.R.; Ribeiro, A.R.; Manfro, R.C. Long-term effects of delayed graft function duration on function and survival of deceased donor kidney transplants. J. Bras. Nefrol. 2019, 41, 231-241. [CrossRef] [PubMed]

6. Metzger, R.A.; Delmonico, F.L.; Feng, S.; Port, F.K.; Wynn, J.J.; Merion, R.M. Expanded criteria donors for kidney transplantation. Am. J. Transplant. 2003, 3 (Suppl. 4), 114-125. [CrossRef]

7. Querard, A.H.; Le Borgne, F.; Dion, A.; Giral, M.; Mourad, G.; Garrigue, V.; Rostaing, L.; Kamar, N.; Loupy, A.; Legendre, C.; et al. Propensity score-based comparison of the graft failure risk between kidney transplant recipients of standard and expanded criteria donor grafts: Toward increasing the pool of marginal donors. Am. J. Transplant. 2018, 18, 1151-1157. [CrossRef]

8. Ojo, A.O.; Hanson, J.A.; Meier-Kriesche, H.; Okechukwu, C.N.; Wolfe, R.A.; Leichtman, A.B.; Agodoa, L.Y.; Kaplan, B.; Port, F.K. Survival in recipients of marginal cadaveric donor kidneys compared with other recipients and wait-listed transplant candidates. J. Am. Soc. Nephrol. 2001, 12, 589-597.

9. Belzer, F.O.; Ashby, B.S.; Dunphy, J.E. 24-hour and 72-hour preservation of canine kidneys. Lancet 1967, 2, 536-538. [CrossRef]

10. Belzer, F.O.; Ashby, B.S.; Gulyassy, P.F.; Powell, M. Successful seventeen-hour preservation and transplantation of human-cadaver kidney. N. Engl. J. Med. 1968, 278, 608-610. [CrossRef]

11. Moers, C.; Smits, J.M.; Maathuis, M.H.; Treckmann, J.; van Gelder, F.; Napieralski, B.P.; Van Kasterop-Kutz, M.; Van Der Heide, J.J.H.; Squifflet, J.-P.; Van Heurn, E.; et al. Machine perfusion or cold storage in deceased-donor kidney transplantation. N. Engl. J. Med. 2009, 360, 7-19. [CrossRef]

12. Zhong, Z.; Lan, J.; Ye, S.; Liu, Z.; Fan, L.; Zhang, Y.; Fu, Z.; Qiao, B.; Ko, D.S.; Wang, Y.; et al. Outcome Improvement for Hypothermic Machine Perfusion Versus Cold Storage for Kidneys From Cardiac Death Donors. Artif. Organs. 2017, 41, 647-653. [CrossRef] [PubMed]

13. Tingle, S.J.; Figueiredo, R.S.; Moir, J.A.; Goodfellow, M.; Talbot, D.; Wilson, C.H. Machine perfusion preservation versus static cold storage for deceased donor kidney transplantation. Cochrane Database Syst. Rev. 2019, 3, CD011671. [CrossRef]

14. Savoye, E.; Macher, M.A.; Videcoq, M.; Gatault, P.; Hazzan, M.; Abboud, I.; Thierry, A.; Bertrand, D.; Drouin, S.; Sayegh, J.; et al. Evaluation of outcomes in renal transplantation with hypothermic machine perfusion for the preservation of kidneys from expanded-criteria donors. Clin. Transplant. 2019, 33, e13536. [CrossRef]

15. Yuan, X.; Theruvath, A.J.; Ge, X.; Floerchinger, B.; Jurisch, A.; García-Cardeña, G.; Tullius, S.G. Machine perfusion or cold storage in organ transplantation: Indication, mechanisms, and future perspectives. Transpl. Int. 2010, 23, 561-570. [CrossRef] [PubMed]

16. Kaths, J.M.; Paul, A.; Robinson, L.A.; Selzner, M. Ex vivo machine perfusion for renal graft preservation. Transplant. Rev. 2018, 32, 1-9. [CrossRef] [PubMed] 
17. von Elm, E.; Altman, D.G.; Egger, M.; Pocock, S.J.; Gøtzsche, P.C.; Vandenbroucke, J.P. The Strengthening the Reporting of Observational Studies in Epidemiology (STROBE) Statement: Guidelines for reporting observational studies. Int. J. Surg. 2014, 12, 1495-1499. [CrossRef] [PubMed]

18. Perico, N.; Cattaneo, D.; Sayegh, M.H.; Remuzzi, G. Delayed graft function in kidney transplantation. Lancet 2004, 364, 1814-1827. [CrossRef]

19. Kienzl-Wagner, K.; Schneiderbauer, S.; Bösmüller, C.; Schneeberger, S.; Pratschke, J.; Ollinger, R. Nighttime procedures are not associated with adverse outcomes in kidney transplantation. Transpl. Int. 2013, 26, 879-885. [CrossRef] [PubMed]

20. Port, F.K.; Bragg-Gresham, J.L.; Metzger, R.A.; Dykstra, D.M.; Gillespie, B.W.; Young, E.W.; Delmonico, F.; Wynn, J.J.; Merion, R.M.; Wolfe, R.A.; et al. Donor characteristics associated with reduced graft survival: An approach to expanding the pool of kidney donors. Transplantation 2002, 74, 1281-1286. [CrossRef]

21. Austin, P.C. Balance diagnostics for comparing the distribution of baseline covariates between treatment groups in propensity-score matched samples. Stat. Med. 2009, 28, 3083-3107. [CrossRef] [PubMed]

22. Schamberger, B.; Lohmann, D.; Sollinger, D.; Stein, R.; Lutz, J. Association of Kidney Donor Risk Index with the Outcome after Kidney Transplantation in the Eurotransplant Senior Program. Ann. Transplant. 2018, 23, 775-781. [CrossRef] [PubMed]

23. Husen, P.C.B.; Davies, L.; Knight, S.; Paul, A.; Ploeg, R.J. End-Hypothermic Machine Perfusion with Oxygenation after Static Cold Storage versus Static Cold Storage alone in ECD Kidneys from Donation after Brain Death Donors: Results of a Prospective International Randomised Controlled Trial in Kidney Transplantation. Available online: https://www.esot.org/resources/consortium-organ-preservation-europe (accessed on 1 June 2020).

24. Bahl, D.; Haddad, Z.; Datoo, A.; Qazi, Y.A. Delayed graft function in kidney transplantation. Curr. Opin. Organ Transplant. 2019, 24, 82-86. [CrossRef] [PubMed]

25. Melih, K.V.; Boynuegri, B.; Mustafa, C.; Nilgun, A. Incidence, Risk Factors, and Outcomes of Delayed Graft Function in Deceased Donor Kidney Transplantation. Transplant. Proc. 2019, 51, 1096-1100. [CrossRef]

26. Tingle, S.J.; Figueiredo, R.S.; Moir, J.A.; Goodfellow, M.; Thompson, E.R.; Ibrahim, I.K.; Bates, L.; Talbot, D.; Wilson, C.H. Hypothermic Machine Perfusion is Superior to Static Cold Storage in Deceased Donor Kidney Transplantation: A Meta-analysis. Clin. Transplant. 2020, e13814. [CrossRef]

27. Gill, J.; Dong, J.; Eng, M.; Landsberg, D.; Gill, J.S. Pulsatile perfusion reduces the risk of delayed graft function in deceased donor kidney transplants, irrespective of donor type and cold ischemic time. Transplantation 2014, 97, 668-674. [CrossRef] [PubMed]

28. Lam, V.W.; Laurence, J.M.; Richardson, A.J.; Pleass, H.C.; Allen, R.D. Hypothermic machine perfusion in deceased donor kidney transplantation: A systematic review. J. Surg. Res. 2013, 180, 176-182. [CrossRef]

29. Kox, J.; Moers, C.; Monbaliu, D.; Strelniece, A.; Treckmann, J.; Jochmans, I.; Leuvenink, H.G.D.; Van Heurn, E.; Pirenne, J.; Paul, A.; et al. The Benefits of Hypothermic Machine Preservation and Short Cold Ischemia Times in Deceased Donor Kidneys. Transplantation 2018, 102, 1344-1350. [CrossRef]

30. Fechner, G.; Pezold, C.; Hauser, S.; Gerhardt, T.; Müller, S.C. Kidney's nightshift, kidney's nightmare? Comparison of daylight and nighttime kidney transplantation: Impact on complications and graft survival. Transplant. Proc. 2008, 40, 1341-1344. [CrossRef]

31. Seow, Y.Y.; Alkari, B.; Dyer, P.; Riad, H. Cold ischemia time, surgeon, time of day, and surgical complications. Transplantation 2004, 77, 1386-1389. [CrossRef]

32. Hoyer, D.P.; Gallinat, A.; Swoboda, S.; Wohlschlaeger, J.; Rauen, U.; Paul, A.; Minor, T. Influence of oxygen concentration during hypothermic machine perfusion on porcine kidneys from donation after circulatory death. Transplantation 2014, 98, 944-950. [CrossRef]

33. Thuillier, R.; Allain, G.; Celhay, O.; Hebrard, W.; Barrou, B.; Badet, L.; Leuvenink, H.G.; Hauet, T. Benefits of active oxygenation during hypothermic machine perfusion of kidneys in a preclinical model of deceased after cardiac death donors. J. Surg. Res. 2013, 184, 1174-1181. [CrossRef] [PubMed] 
34. Darius, T.; Vergauwen, M.; Smith, T.; Gerin, I.; Joris, V.; Mueller, M.; Aydin, S.; Muller, X.; Schlegel, A.; Nath, J.; et al. Brief $\mathrm{O}^{2}$ uploading during continuous hypothermic machine perfusion is simple yet effective oxygenation method to improve initial kidney function in a porcine autotransplant model. Am. J. Transplant. 2020. [CrossRef] [PubMed]

35. Jochmans, I.; Hofker, H.S.; Davies, L.; Knight, S.; Pirenne, J.; Ploeg, R.J. Oxygenated Hypothermic Machine Perfusion of Kidneys Donated after Circulatory Death: An International Randomised Controlled Trial [abstract]. Am. J. Transplant. 2019, 19 (Suppl. 3), 312. Available online: https://atcmeetingabstracts.com/abstract/oxygenated-hypothermic-machine-perfusion-of-kidneysdonated-after-circulatory-death-an-international-randomised-controlled-trial/ (accessed on 20 August 2019).

(C) 2020 by the authors. Licensee MDPI, Basel, Switzerland. This article is an open access article distributed under the terms and conditions of the Creative Commons Attribution (CC BY) license (http://creativecommons.org/licenses/by/4.0/). 